\title{
Ocular haemodynamics and colour contrast sensitivity in patients with type 1 diabetes
}

Oliver Findl, Susanne Dallinger, Birgit Rami, Kaija Polak, Edith Schober, Andreas Wedrich, Eva Ries, Hans-Georg Eichler, Michael Wolzt, Leopold Schmetterer

\begin{abstract}
Background-There is evidence that altered ocular blood flow is involved in the development and progression of diabetic retinopathy. However, the nature of these perfusion abnormalities is still a matter of controversy. Ocular haemodynamics were characterised with two recently introduced methods.

Methods-The cross sectional study was performed in 59 patients with type 1 diabetes with a diabetes duration between 12 and 17 years and an age less than 32 years and a group of 25 age matched healthy controls. Scanning laser Doppler flowmetry and laser interferometric measurement of fundus pulsation amplitude were used to assess retinal and pulsatile choroidal blood flow, respectively. In addition, colour contrast sensitivity along the tritan axis was determined.

Results-Fundus pulsation amplitude, but not retinal blood flow, increased with the progression of diabetic retinopathy. Retinal blood flow was influenced by plasma glucose levels $(r=0.32)$, whereas fundus pulsation amplitude was associated with $\mathbf{H b A}_{1 \mathrm{c}}(r=0.30)$. In addition, a negative correlation between the colour contrast sensitivity along the tritan axis and retinal blood flow was observed.

Conclusions-The present study indicates that pulsatile choroidal blood flow increases with the progression of diabetic retinopathy. Increased retinal blood flow appears to be related to loss of colour sensitivity in patents with type 1 diabetes.

(Br f Ophthalmol 2000;84:493-498)
\end{abstract}

Department of

Ophthalmology

O Findl

A Wedrich

E Ries

Department of

Pediatrics

B Rami

E Schober

Institute of Medical

Physics

L Schmetterer

Correspondence to: Dr L Schmetterer, Department of Clinical

Pharmacology, Währinger

Gürtel 18-20, A-1090

Vienna, Austria

leopold.schmetterer@

univie.ac.at

Accepted for publication 13 January 2000

Altered ocular blood flow may contribute to the development and progression of diabetic retinopathy. However, the exact nature of ocular blood flow abnormalities in diabetes has not yet been established and the results obtained in patients with type 1 diabetes and type 2 diabetes strongly depend on the technique used for the assessment of ocular haemodynamics. Both, increased ${ }^{1-3}$ and decreased retinal blood flow ${ }^{4}$ have been observed in patients with diabetes compared with healthy controls. A recent study using laser Doppler velocimetry for the assessment of retinal blood flow indicates that retinal perfusion is already increased in patients with type 1 diabetes before the clinical onset of diabetic retinopathy. ${ }^{6}$ This observation has further supported the concept that increased retinal blood flow may have a key role in the development of diabetic retinopathy. ${ }^{78}$

The purpose of the present study was to determine whether retinal and choroidal blood flow are altered in the early stages of diabetic retinopathy. Retinal blood flow was assessed with scanning laser Doppler flowmetry. ${ }^{9}$ Pulsatile choroidal blood flow was assessed with laser interferometric measurement of fundus pulsation. ${ }^{10}$ Ocular haemodynamic factors were compared with the results of colour contrast sensitivity testing. This was done in an effort to establish a relation between alterations in ocular haemodynamics and loss of colour contrast sensitivity. In order to minimise the effect of potentially confounding factors such as duration of diabetes or age we focused on a study population with type 1 diabetes with a duration of 12-17 years and an age of less than 32 years.

\section{Methods}

PATIENTS

After approval from the ethics committee of Vienna University School of Medicine, 59 subjects (39 males, 20 females) with insulin dependent diabetes mellitus (age range 13-31 years, mean 23.1 (SD 4.3) years) and 25 healthy control subjects (17 males, eight females) were studied (age range 14-32 years; mean 23.8 (4.0) years). The nature of the study was explained and all subjects gave written consent to participate. Patients with type 1 diabetes with a disease duration between 12 and 17 years were included in the present study. Exclusion criteria were systemic hypertension (defined as systolic blood pressure $>145 \mathrm{~mm} \mathrm{Hg}$, diastolic blood pressure $>90$ $\mathrm{mm} \mathrm{Hg}$ ), or any sign of non-diabetes induced vascular complications.
In all subjects the left eyes were studied. Best corrected visual acuity was determined with Early Treatment Diabetic Retinopathy Study (ETDRS) acuity charts. ${ }^{11}$ Ophthalmic examination included slit lamp biomicroscopy and indirect funduscopy. In addition, colour fundus photography of seven fields was performed and the eyes were classified according to the Modified Airlie House Classification. ${ }^{12}{ }^{13}$ Subjects were excluded if any ocular disease except diabetic retinopathy was evident at the prestudy ophthalmic examination.

All ocular haemodynamic variables were measured twice within 5 minutes. To ensure a sufficient test/retest reproducibility only those variables with an intraindividual coefficient of variation of less than $20 \%$ were included for analysis. 
Table 1 Characteristics and diabetic retinopathy stages * of subjects with type 1 diabetes

\begin{tabular}{|c|c|c|c|}
\hline & Level 1 & Level 2 & Level 3 \\
\hline Number & 20 & 27 & 12 \\
\hline Age (years) & $22.9(4.2)$ & $23.7(4.5)$ & $22.1(4.0)$ \\
\hline Glucose plasma levels $(\mathrm{mg} / 100 \mathrm{ml})$ & $121.3(57.9)$ & $164.3(67.0)$ & $194.8(84.4)$ \\
\hline $\mathrm{HbA}_{1 \mathrm{c}}(\%)$ & $7.7(1.1)$ & $8.5(1.0)$ & $10.7(1.5)$ \\
\hline Systolic blood pressure $(\mathrm{mm} \mathrm{Hg})$ & $127.9(7.1)$ & $121.1(10.5)$ & $124.8(8.0)$ \\
\hline Diastolic blood pressure $(\mathrm{mm} \mathrm{Hg})$ & $66.3(12.1)$ & $60.6(10.1)$ & $60.0(7.3)$ \\
\hline Pulse rate (min) & $72.4(11.4)$ & $73.4(11.2)$ & $74.3(10.5)$ \\
\hline
\end{tabular}

^According to Modified Airlie House Classification (level 1: no retinopathy, level 2: microaneurysms (one or more) only, level 3: microaneurysms and one or more of the following: retinal haemorrhages, but total haemorrhages and microaneurysms $(\mathrm{H} / \mathrm{Ma})$ less than standard photo No 2; hard exudates (HE) <standard photo No 3; soft exudates (SE) questionably present; intraretinal microvascular abnormalities (IRMA) questionably present, venous beading (VB) questionably present or venous focal narrowing or loops definitely present.

\section{Methods}

SCANNING LASER DOPPLER FLOWMETRY

Retinal microcirculation was assessed with a commercially available scanning laser Doppler flowmeter (Heidelberg Retina Flowmeter, HRF, Heidelberg Engineering, Heidelberg, Germany $\left.{ }^{914}\right)$. This system combines laser Doppler flowmetry with laser scanning tomography. Briefly, the vascularised tissue is illuminated by coherent laser light. Scattering on moving red blood cells (RBCs) leads to a frequency shift in the scattered light. In contrast, static scatterers in tissue do not change light frequency, but lead to randomisation of light directions impinging on RBCs. This light diffusing in vascularised tissue leads to a broadening of the spectrum of scattered light, from which mean RBC velocity, the blood volume, and the blood flow (flow) can be calculated. These variables are calculated from the backscattered light for each point during the scanning process and a two dimensional map of retinal perfusion is created. Hence, these factors can be quantified in relative units for any image point. In the present study $20 \times$ 20 pixel areas were chosen for calculation of haemodynamic variables $(200 \times 200 \mu \mathrm{m})$. This area was located temporal of the fovea. Care
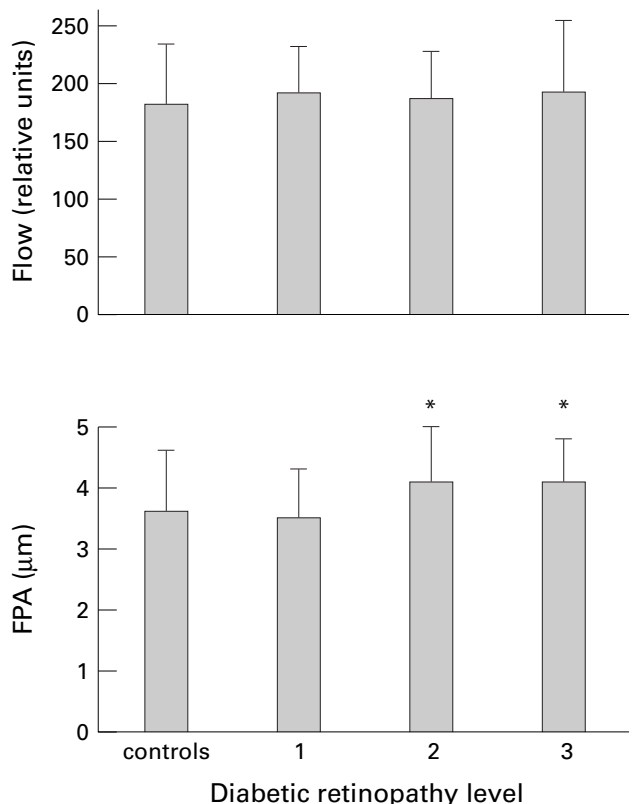

was taken that it did not overlap with the avascular retinal zone. Two images were taken from each subject within 5 minutes in order to assess the short term variability of the measurements. The reproducibility of this method in healthy subject has been reported previously. ${ }^{15}$

\section{FUNDUS PULSATIONS}

Pulse synchronous pulsations of the ocular fundus were measured with laser interferometry. The method is described in detail by Schmetterer $e$ t al. ${ }^{10}$ Briefly, the eye is illuminated by the beam of a single mode laser diode with a wavelength $(\lambda)$ of $783 \mathrm{~nm}$. The laser light is reflected at both the front surface of the cornea and at the retinal pigment epithelium. The two re-emitted waves produce interference fringes from which the distance changes between cornea and retina during a cardiac cycle can be calculated. Distance changes between cornea and retina lead to a corresponding variation of the interference order $(\Delta N(t))$. This change in interference order can be evaluated by counting the fringes moving inwards and outwards during the cardiac cycle. Changes in optical distance $(\Delta \mathrm{L}(\mathrm{t}))$, corresponding to the cornea-retina distance changes, can then be calculated by $\Delta \mathrm{L}(\mathrm{t})$ $=\Delta \mathrm{N}(\mathrm{t}) \cdot \lambda / 2$. The maximum distance change is called fundus pulsation amplitude (FPA) and estimates the local pulsatile blood flow. ${ }^{16}$ To obtain information on the choroidal blood flow the macula, where the retina lacks vasculature, was chosen for measurements. Two measurements were taken from each subject within 5 minutes in order to assess the short term variability. The reproducibility of this method in healthy subjects has been reported previously. ${ }^{16}{ }^{17}$

DOPPLER SONOGRAPHY

Blood flow velocities in the ophthalmic artery were assessed using Doppler ultrasound. ${ }^{18}$ The
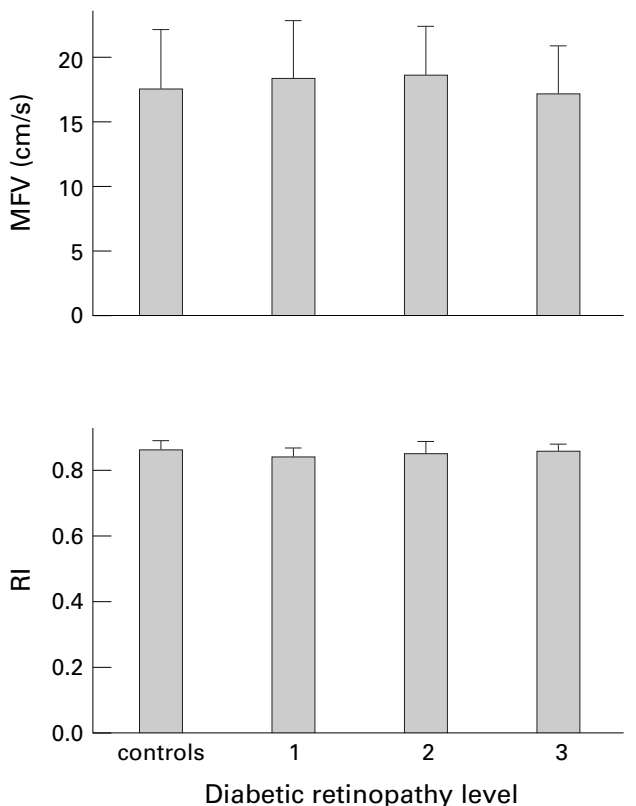

Figure 1 Ocular haemodynamic variables in the different stages of diabetic retinopathy and in healthy controls. Retinopathy stages are given according to Modified Airlie House Classification (for definition see Table 1). Asterisks indicate significant differences between the different stages of retinopathy. (Flow = retinal blood flow, FPA = fundus pulsation amplitude, $M F V=$ mean flow velocity, $R I=$ resistive index). Data are presented as means (SD). 

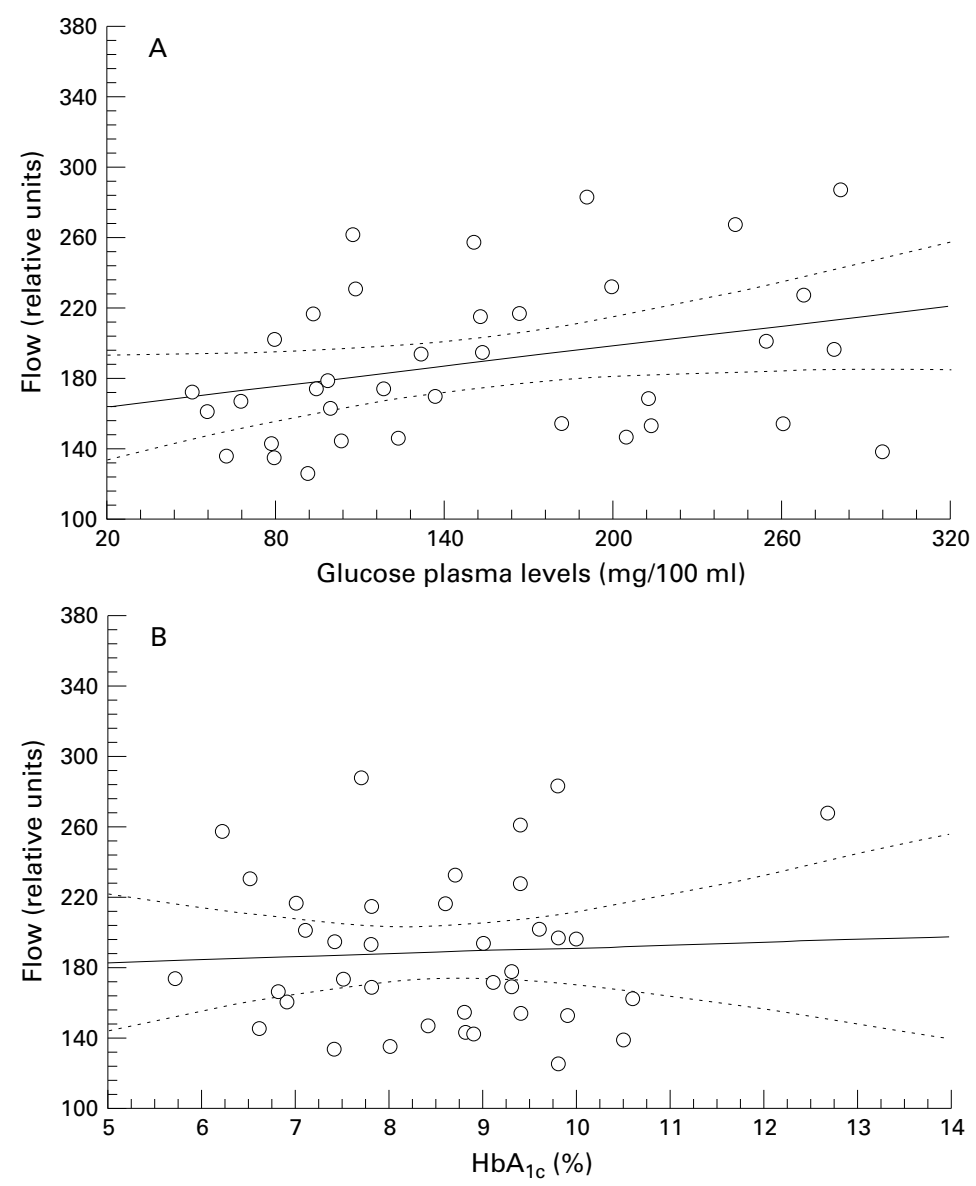

Figure 2 Linear correlation between retinal blood flow (flow) and glucose plasma levels $(A)$ and $\mathrm{Hb}_{1 c}$ (B). The broken lines represent the $95 \%$ confidence intervals.

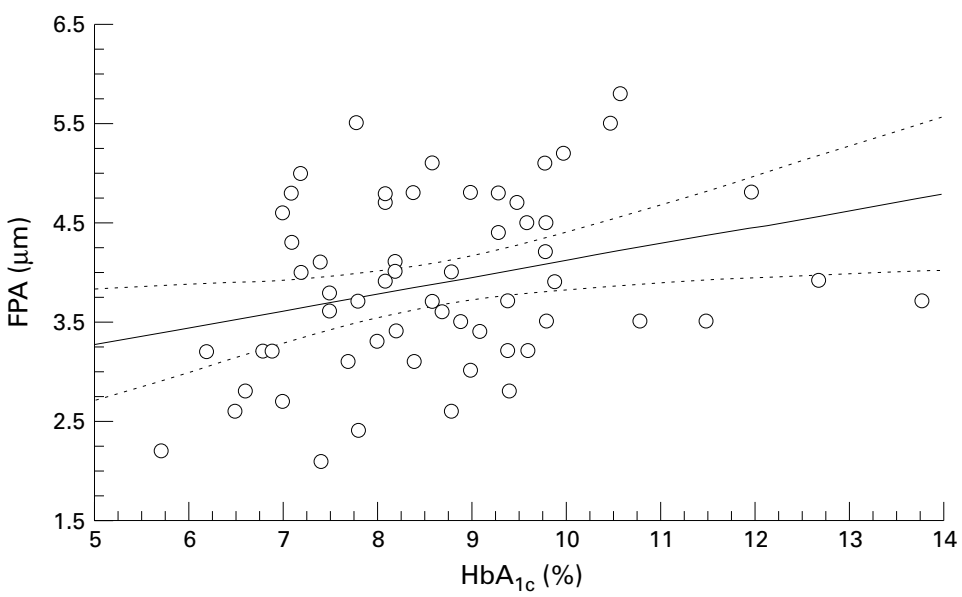

Figure 3 Linear correlation between fundus pulsation amplitude (FPA) and $\mathrm{Hb} \mathrm{A}_{1 \mathrm{c}}$. The broken lines represent the $95 \%$ confidence intervals.

ophthalmic artery was measured anteriorly, at the point where it crosses the optic nerve, with a 7.5 MHZ probe (CFM 750, Vingmed Sound, Horten, Norway). Mean flow velocity (MFV) was calculated as time mean of the spectral outline. Resistivity index (RI) was calculated as $\mathrm{RI}=$ (peak systolic velocity - end diastolic velocity)/peak systolic velocity.

COLOUR CONTRAST SENSITIVITY

Colour contrast sensitivity along the tritan axis was investigated in order to evaluate the prop-
Table 2 p Values of multiple regression analysis between ocular haemodynamics and age, systolic and diastolic blood pressure, pulse rate, intraocular pressure, glucose plasma levels, and $\mathrm{Hb} \mathrm{A}_{1}$

\begin{tabular}{lll}
\hline & $\begin{array}{l}\text { Retinal blood } \\
\text { flow }\end{array}$ & $\begin{array}{l}\text { Fundus pulsation } \\
\text { amplitude }\end{array}$ \\
\hline Age & 0.51 & 0.65 \\
Systolic blood pressure & 0.49 & 0.76 \\
Diastolic blood pressure & 0.45 & 0.83 \\
Pulse rate & 0.90 & 0.41 \\
Intraocular pressure & 0.68 & 0.43 \\
Glucose plasma levels & 0.03 & 0.17 \\
HbA $_{1 \mathrm{c}}$ & 0.54 & 0.03 \\
\hline
\end{tabular}

erties of colour vision. ${ }^{19}$ The system comprises a computer graphics device and a colour monitor system. The threshold chrominance of a coloured optotype without changes in luminance compared with the surrounding is determined. This method is more sensitive than other colour vision tests available. ${ }^{19}$

APPLANATION TONOMETRY

Intraocular pressure (IOP) was measured by Goldmann applanation tonometry (HaagStreit, Bern, Switzerland).

\section{LABORATORY ANALYSIS}

Glucose and $\mathrm{HbA}_{1 \mathrm{c}}$ plasma concentrations were measured by routine procedures.

NON-INVASIVE MEASUREMENT OF SYSTEMIC

HAEMODYNAMICS

Systolic and diastolic blood pressure (SBP, DBP) were measured on the upper arm by an automated oscillometric device. Mean arterial pressure (MAP) was calculated as $1 / 3 \mathrm{SBP}+2 / 3$ DBP. Pulse rate (PR) was automatically recorded from a finger pulse oximetric device (HP-CMS patient monitor, Hewlett Packard, Palo Alto, CA, USA).

\section{DATA ANALYSES}

Ocular haemodynamics and colour contrast sensitivity thresholds in the different stages of diabetic retinopathy and the healthy controls were compared with ANOVA. Linear regression analysis was performed to determine the correlation between glucose and $\mathrm{HbA}_{1 \mathrm{c}}$ plasma levels and ocular haemodynamics in patients with diabetes. The association between colour contrast sensitivity thresholds and ocular haemodynamic variables was also investigated with linear regression. Multiple regression analysis was performed in order to characterise determinants of retinal blood flow and fundus pulsation amplitude. Data are presented as means (SD). The level of significance was set as $\mathrm{p}=0.05$.

\section{Results}

Characteristics of patients with type 1 diabetes are given in Table 1 . The stage of retinopathy was not dependent on age, systemic blood pressure, or pulse rate. By contrast plasma glucose levels $(\mathrm{p}=0.012)$ and $\mathrm{HbA}_{1 \mathrm{c}}$ values ( $\mathrm{p}$ $<0.001$ ) were higher in the more advanced stages of diabetic retinopathy. Best corrected visual acuity was $20 / 20$ in all subjects with diabetic retinopathy levels 1 and 2 in both eyes. In 
Table 3 Colour contrast sensitivity thresholds along the tritan axis in patients with different stages of retinopathy ${ }^{\star}$

\begin{tabular}{llllll}
\hline Diabetic retinopathy level & Controls & Level 1 & Level 2 & Level 3 & \\
\hline Number & 25 & 20 & 27 & 12 & \\
Threshold (\%) & $7.1(1.7)$ & $7.3(1.9)$ & $8.1(2.1)$ & $10.1(3.0)$ & $\mathrm{p}=0.020$ \\
\hline
\end{tabular}

^According to Modified Airlie House Classification, for definition see Table 1.

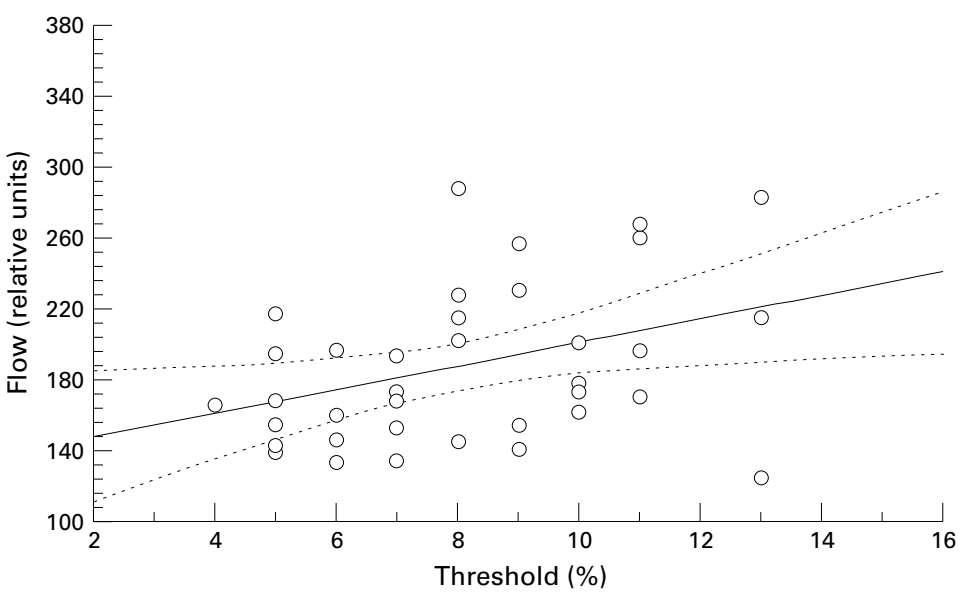

Figure 4 Linear correlation between retinal blood flow (flow) and threshold values from colour vision testing. The broken lines represent the $95 \%$ confidence intervals.

the eyes with diabetic retinopathy level 3 seven patients had a best corrected visual acuity of $20 / 20$ and the remaining five subjects had $20 / 25$.

We were able to obtain scanning laser Doppler flowmetry recordings with a coefficient of variation less than $20 \%$ in 36 eyes of patients with type 1 diabetes and 22 eyes of healthy control subjects. The retinal flow values as obtained with this technique were not dependent on the stage of retinopathy and were comparable between patients with type 1 diabetes and healthy control subjects (Fig 1). By contrast, FPA increased with increasing stage of the disease $(p=0.049$, Fig 1$)$. All fundus pulsation measurements had a coefficient of variation less than 20\%. Doppler sonographic measurements of blood flow velocities in the ophthalmic artery fulfilled this criterion in all eyes. Neither MFV in the ophthalmic artery nor RI was dependent on the stage of retinopathy.

Retinal blood flow was associated with plasma glucose levels $(r=0.32, \mathrm{p}=0.048$, Fig $2)$. By contrast, retinal flow values were independent of $\mathrm{HbA}_{1 \mathrm{c}}(r=0.06, \mathrm{p}=0.74)$. FPA was associated with $\mathrm{HbA}_{1 \mathrm{c}}(r=0.30, \mathrm{p}=$ 0.021 , Fig 3), but was independent of plasma glucose levels $(r=-0.05, \mathrm{p}=0.72)$. The results of multiple regression analysis are presented in Table 2. Again these analyses indicate that retinal blood flow is dependent on glucose plasma levels, whereas FPA is dependent on $\mathrm{HbA}_{1 \mathrm{c}}$. The other variables had no significant impact on ocular haemodynamics.

The threshold along the tritan axis was dependent on the stage of retinopathy (Table $3 ; p=0.020)$. The results of the comparisons between ocular haemodynamics and colour contrast sensitivity testing are shown in Figure 4. Thresholds were significantly associated with retinal flow values $(r=0.38, \mathrm{p}=0.020$,
Fig 4). This indicates a negative association between retinal blood flow and colour contrast sensitivity. By contrast, neither haemodynamic variables in the choroid nor in the ophthalmic artery were correlated with colour vision.

\section{Discussion}

A variety of studies have focused on retinal blood flow in diabetic retinopathy. The results are, however, strongly dependent on the methods employed. In the present study we used scanning laser Doppler flowmetry in order to assess retinal haemodynamics. In keeping with a laser Doppler velocimetry study ${ }^{20}$ we did not observe a difference between retinal blood flow in patients with early stages of diabetic retinopathy and patients with no retinopathy. Retinal blood flow in patients with diabetes was not different compared with healthy controls in the present study. A recent study using laser Doppler velocimetry reported that retinal blood flow is already increased in diabetic subjects with no clinical signs of retinopathy compared with healthy controls. ${ }^{6}$

The present study reveals that glucose plasma levels are a determinant of retinal blood flow. This could well contribute to previous contradictory findings concerning retinal blood flow in the different stages of diabetic retinopathy. The fact that glucose induces retinal vasodilatation has been reported previously using a variety of techniques for the assessment of retinal blood flow. ${ }^{52}{ }^{22} \mathrm{HbA}_{1 \mathrm{c}}$ was not identified as an independent determinant of retinal blood flow in the present study, which is in contrast with previous data using fluorescein angiography for the assessment of retinal blood flow. ${ }^{23}$ The use of retinal circulation times as measured from angiograms in the study of diabetes induced retinal blood flow abnormalities is, however, still controversial and several limitations of the method have been pointed out. ${ }^{824}$

Ocular FPA, assessing pulsatile choroidal blood flow, was dependent on the stage of diabetic retinopathy, indicating that choroidal blood flow is increased in mild and moderate retinopathy. This is in keeping with several studies in STZ induced diabetic rats $^{25-27}$ and with a recent study using pneumotonometry for the assessment of pulsatile ocular blood flow. ${ }^{28}$ However, in a previous study employing laser interferometry we did not find increased FPA in the early stages of diabetic retinopathy. ${ }^{29}$ Several differences between this previous cross sectional trial and the present study exist, which could explain this discrepancy. In the present study only patients with type 1 diabetes were included, whereas our former study population consisted of patients with type 1 and type 2 diabetes. In addition, the present study focused on young patients with no concomitant vascular diseases, whereas our former study population consisted of patients with a mean age of approximately 60 years. This is of importance, because an age dependency of pulsatile ocular blood flow has been reported. ${ }^{30} 31$

In contrast with retinal blood flow $\mathrm{HbA}_{1}$ was a determinant of pulsatile choroidal blood 
flow. This indicates that choroidal blood flow is more dependent on metabolic control than on actual glucose plasma levels. Until now little attention has been drawn to choroidal blood flow abnormalities in the pathogenesis of diabetic retinopathy. However, recent high resolution histological studies indicate that diabetes induces severe alterations in choroidal microcirculation. ${ }^{32}$ The reason for this early increase in pulsatile choroidal blood flow remains to be established. An altered endothelial function, as evidenced from an abnormal choroidal vascular response to a nitric oxidesynthase inhibitor, ${ }^{33}$ could well contribute to choroidal overperfusion. Glucose plasma levels had no significant impact on choroidal blood flow.

A key finding of the present study is that retinal blood flow was negatively correlated with colour contrast sensitivity along the tritan axis. To the best of our knowledge this is the first report to show an association between retinal perfusion and colour visual function. The reason for this negative correlation remains to be elucidated. Our findings are, however, compatible with a recent study showing that systemic hyperoxia improves contrast sensitivity in patients with early diabetic retinopathy. ${ }^{34}$ Whereas the authors ascribe their observation to the reversal of retinal tissue hypoxia during oxygen breathing a beneficial effect of hyperoxia induced reduction in retinal blood flow should also be considered. Whether a reduction in retinal blood flow itself may improve visual function in early diabetic retinopathy should be further investigated.

In contrast with the choroidal haemodynamics, variables in the ophthalmic artery were not dependent on the stage of diabetic retinopathy. Previous studies indicate that blood flow velocities in this vessel are slightly reduced in diabetic subjects compared with healthy controls. ${ }^{35}$ This does, however, not mean that volumetric blood flow in the ophthalmic artery is reduced, but may rather reflect vasodilatation in early diabetes. ${ }^{8}$

Assessment of retinal and choroidal haemodynamics with scanning laser Doppler flowmetry and topical fundus pulsation measurement has some limitations. The reproducibility of scanning laser Doppler flowmetry recordings is of critical importance. We have already reported previously that reproducible results cannot be obtained in all healthy subjects under study. ${ }^{15}$ The present study extends these findings in patients with diabetic retinopathy. Test/retest variability smaller than $20 \%$ was observed in less than $70 \%$ of the eyes under study. An additional limitation arises from the principle of laser Doppler flowmetry. Flow readings are not only dependent on the blood flow in the measurement volume, but also on the scattering properties of the surrounding tissue. Owing to the morphological changes associated with diabetic retinopathy it is not completely clear whether readings as obtained with scanning laser Doppler flowmetry can be compared in the different stages of the disease.

With laser interferometric measurement of fundus pulsation it is clear that only the pulsa- tile component of blood flow is assessed. Obviously conclusions on total choroidal blood flow are critically dependent on changes in flow pulsatility in the diabetic choroid. ${ }^{36}$ The present study indicates choroidal vasodilatation in the early stages of diabetic retinopathy. A decrease in choroidal vascular resistance would, however, lead to a reduction in flow pulsatility. Hence FPA rather underestimates the effect of diabetic retinopathy on choroidal blood flow in the present study.

In conclusion, our results in patients with type 1 diabetes indicate that choroidal, but not retinal blood flow, is elevated in the early stages of diabetic retinopathy compared with diabetic patients without retinopathy and healthy control subjects. Whereas retinal blood flow is associated with actual glucose plasma levels, choroidal blood flow depends on metabolic control.

Financial support from Jubiläumsfonds der Österreichischen Nationalbank (Grant No 5940/4) is acknowledged.

1 Kohner EM, Hamilton AM, Saunders SJ, et al. The retinal blood flow in diabetes. Diabetologia 1975;11:27-33.

2 Grunwald JE, Riva CE, Baine J, et al. Total retinal volumetric blood flow rate in diabetic patients with poor glycemic control. Invest Ophthalmol Vis Sci 1992;33:356-63.

3 Patel V, Rassam S, Newsom R, et al. Retinal blood flow in diabetic retinopathy. BMF 1992;305:678-83.

4 Wolf S, Arend O, Toonen H, et al. Retinal capillary blood flow measurement with a scanning laser ophthalmoscope. Ophthalmology 1991;98:996-1000.

5 Bursell SE, Clermont AC, Kinsley BT, et al. Retinal blood flow changes in patients with insulin-dependent diabetes mellitus and no diabetic retinopathy. Invest Ophthalmol Vis Sci 1996;37:886-97.

6 Grunwald JE, DuPont J, Riva CE. Retinal haemodynamics in patients with early diabetes mellitus. $\mathrm{Br} \mathcal{F}$ Ophthalmol 1996;80:327-31.

7 Kohner EM, Patel V, Rassam SMB. Role of blood flow and impaired autoregulation in the pathogenesis of diabetic retinopathy. Diabetes 1995;44:603-7.

8 Schmetterer L, Wolzt M. Ocular blood flow and associated functional deviations in diabetic retinopathy. Diabetologia 1999;42:387-405.

9 Michelson G, Langhans MJ, Groh MJM. Clinical investigation of the combination of a scanning laser ophthalmoscope and laser Doppler flowmeter. Ger $\mathcal{F}$ Ophthalmol 1995;4:342-9

10 Schmetterer L, Lexer F, Unfried C, et al. Topical measurement of fundus pulsations. Opt Eng 1995;34:71116.

11 Klein R, Klein BE, Moss SE, et al. Inter-observed variation in refraction and visual acuity measurements using a standardized protocol. Ophthalmology 1983;90:1357-9.

12 The Diabetic Retinopathy Study Group. Report No 7. A modification of the Airlie House classification of diabetic retinopathy. Invest Ophthalmol Vis Sci 1981;21:211-26.

13 Klein BE, Davis MD, Segal P, et al. Diabetic retinopathy. Assessment of severity and progression. Ophthalmology 1984;91:10-17.

14 Michelson G, Schmauss B, Langhans MJ, et al. Principle, validity, and reliability of scanning laser Doppler flowmetry. F Glaucoma 1996;5:99-105.

15 Strenn K, Menapace R, Rainer G, et al. Reproducibility and sensitivity of scanning laser Doppler flowmetry during graded changes in $\mathrm{pO}_{2}$. Br f Ophthalmol 1997;81:360-4.

16 Schmetterer L, Dallinger S, Findl O, et al. Noninvasive investigations of the normal ocular circulation. Invest Ophthalmol Vis Sci 1998;39:1210-20.

17 Schmetterer L, Strenn K, Findl O, et al. Effects of antiglaucoma drugs on ocular haemodynamics in healthy voluncoma drugs on ocular haemodynamics in

18 Lieb WE, Cohen SM, Merton DA, et al. Color Doppler maging of the eye and the orbit. Arch Ophthalmol 1991;109:527-31

19 Gündüz K, Arden GB, Perry S, et al. Color vision defects in ocular hypertension and glaucoma. Quantification with a computer-driven color television system. Arch Ophthalmol 1998;106:929-35.

20 Grunwald JE, Riva CE, Sinclair SH, et al. Laser Doppler velocimetry study of retinal circulation in diabetes mellitus. Arch Ophthalmol 1986;104:991-6.

21 Atherton A, Hill DW, Keen H, et al. The effect of acute hyperglycaemia on the retinal circulation of the normal cat. Diabetologia 1996;18:233-7.

22 Grunwald JE, Riva CE, Martin DB, et al. Effect of an insulin-induced decrease in blood glucose on the human diabetic retinal circulation. Ophthalmology 1987;94:161420 
23 Bertram B, Wolf S, Arend O, et al. Retinale Durchblutung und aktueller Blutzuckerwert bei Retinopathia diabetica. und aktueller Blutzuckerwert bei Retinop

24 Kohner EM. The problems of retinal blood flow in diabetes. Diabetes 1976;25(Suppl 2):839-44.

25 Pugliese G, Tilton RG, Speedy A, et al. Effects of very mild versus overt diabetes on vascular haemodynamics and barrier function in rats. Diabetologia 1989;32:845-57.

26 Sutera SP, Chang K, Marvel J, et al. Concurrent increases in regional hematocrit and blood flow in diabetic rats: prevention by sorbinil. Am f Physiol 1992;263:H945-50.

27 Tilton RG, Chang K, Pugliese G, E et al. Prevention of haemodynamic and vascular albumin filtration changes in diabetic rats by aldose reductase inhibitors. Diabetes 1989 37:1258-70.

28 MacKinnon JR, O'Brien C, Swa K, et al. Pulsatile ocular blood flow in untreated diabetic retinopathy. Acta Ophthalmol 1997;75:661-4.

29 Schmetterer L, Salomon A, Rheinberger A, et al. Fundus pulsation measurement in diabetic retinopathy. Graefes Arch Clin Exp Ophthalmol 1997;235:283-7.
30 Ravalico G, Toffoli G, Pastori G, et al. Age-related ocular blood flow changes. Invest Ophthalmol Vis Sci 1996;37: blood flow

31 Dallinger S, Findl O, Strenn K, et al. Age dependence of choroidal blood flow. $\mathcal{F}$ Am Geriatr Soc 1998;46:484-7.

$32 \mathrm{Mc}$ Leod DS, Lutty GA. High-resolution histologic analysis of the human choroidal vasculature. Invest Ophthalmol Vis Sci 1994;35:3799-811.

33 Schmetterer L, Findl O, Fasching P, et al. Nitric oxide and ocular blood flow in patients with IDDM. Diabetes 1997;46:653-8.

34 Harris A, Arend O, Danis RP, et al. Hyperoxia improves contrast sensitivity in early diabetic retinopathy. $\mathrm{Br} f \mathrm{Oph}$ thalmol 1996;80:209-13.

35 Tamaki Y, Nagahara M, Yamashita $H$, et al. Blood velocity in the ophthalmic artery determined by color Doppler imaging in normal subjects and diabetics. Fpn 7 Ophthalmol ing in normal subj

36 Krejcy K, Wolzt M, Kreuzer C, et al. Characterisation of angiotensin-II effects on cerebral and ocular circulation by angiotensin-II effects on cerebral and ocular circulation by
noninvasive methods. Br f Clin Pharmacol 1997;43:501-8. 